\title{
Linear time equivalence of Littlewood-Richardson coefficient symmetry maps
}

\author{
Olga Azenhas, Alessandro Conflitti and Ricardo Mamede $\|^{\dagger}$ \\ CMUC, Centre for Mathematics, University of Coimbra, Apartado 3008, 3001-454 Coimbra, Portugal. \\ E-mail: \{oazenhas,conflitt,mamede\}@mat.uc.pt
}

\begin{abstract}
Benkart, Sottile, and Stroomer have completely characterized by Knuth and dual Knuth equivalence a bijective proof of the Littlewood-Richardson coefficient conjugation symmetry, i.e. $c_{\mu, \nu}^{\lambda}=c_{\mu^{t}, \nu^{t}}^{\lambda^{t}}$. Tableau-switching provides an algorithm to produce such a bijective proof. Fulton has shown that the White and the Hanlon-Sundaram maps are versions of that bijection. In this paper one exhibits explicitly the Yamanouchi word produced by that conjugation symmetry map which on its turn leads to a new and very natural version of the same map already considered independently. A consequence of this latter construction is that using notions of Relative Computational Complexity we are allowed to show that this conjugation symmetry map is linear time reducible to the Schützenberger involution and reciprocally. Thus the Benkart-Sottile-Stroomer conjugation symmetry map with the two mentioned versions, the three versions of the commutative symmetry map, and Schützenberger involution, are linear time reducible to each other. This answers a question posed by Pak and Vallejo.

Résumé. Benkart, Sottile, et Stroomer ont complètement caractérisé par équivalence et équivalence duelle à Knuth une preuve bijective de la symétrie de la conjugaison des coefficients de Littlewood-Richardson, i.e. $c_{\mu, \nu}^{\lambda}=c_{\mu^{t}, \nu^{t}}^{\lambda^{t}}$. Le tableau-switching donne un algorithme par produire une telle preuve bijective. Fulton a montré que les bijections de White et de Hanlon et Sundaram sont des versions de celle bijection. Dans ce papier on exhibe explicitement le mot de Yamanouchi produit par cette bijection de conjugaison lequel à son tour conduit à une nouvelle version très naturelle de la même bijection déjà considérée indépendantement. Une conséquence de cette dernière construction c'est que en utilisant des notions de Complexité Computationnelle Relative nous pouvons montrer que cette bijection de symétrie de la conjugaison est linéairement réductible à la involution de Schützenberger et réciproquement. À cette cause la bijection de symétrie de la conjugaison de Benkart, Sottile et Stroomer avec les deux versions mentionnées, aussi bien les trois versions de la bijection de la commutativité, et la involution de Schützenberger sont linéairement réductibles à chacune de les autres. Ça répond à une question posée par Pak et Vallejo.
\end{abstract}

Keywords: Symmetry maps of Littlewood-Richardson coefficients; conjugation symmetry map; linearly time reduction of Young tableaux bijections; tableau-switching; Schützenberger involution.

\footnotetext{
${ }^{\dagger}$ The three authors are supported by CMUC - Centro de Matemática da Universidade Coimbra. The second author is also supported by FCT Portuguese Foundation of Science and Technology (Fundação para a Ciência e a Tecnologia) Grant SFRH/BPD/30471/2006.
}

1365-8050 @ 2009 Discrete Mathematics and Theoretical Computer Science (DMTCS), Nancy, France 


\section{Introduction}

Given partitions $\mu$ and $\nu$, the product $s_{\mu} s_{\nu}$ of the corresponding Schur functions is a non-negative integral linear combination of Schur functions

$$
s_{\mu} s_{\nu}=\sum_{\lambda} c_{\mu \nu}^{\lambda} s_{\lambda},
$$

where $c_{\mu \nu}^{\lambda}$ is called the Littlewood-Richardson coefficient (LiRi; $\left.\mathbf{M a c}, \mathbf{S a} ; \mathbf{S t}\right)$. Let $\lambda^{t}$ denote the conjugate or transpose of the partition $\lambda$. It is obvious from the commutativity of multiplication that $c_{\mu \nu}^{\lambda}=c_{\nu \mu}^{\lambda}$, called the commutativity symmetry, and it is less obvious the conjugation symmetry $c_{\mu \nu}^{\lambda}=c_{\mu^{t} \nu^{t}}^{\lambda^{t}}$. As there are several Littlewood-Richardson rules to compute these numbers, the combinatorics of their symmetries is quite intriguing since in all of them the commutativity is hidden, and the conjugation is either hidden or partially hidden (BZ, KT, PV1). This is in contrast with the fact that most of the symmetries are explicitly exhibited by simple means (PV1). By "hidden" or "simple" we are referring to the computational complexity of the operations needed to reveal such symmetries. Let $\operatorname{LR}(\lambda / \mu, \nu)$ be the set of Littlewood-Richardson (LR for short) tableaux (LiRi) of shape $\lambda / \mu$ and content $\nu$. Then $c_{\mu \nu}^{\lambda}$ counts the number of elements of this set. If one writes $c_{\mu \nu}^{\lambda}=: c_{\mu \nu \lambda}$, with $\lambda^{\vee}$ the complement partition of $\lambda$ regarding some rectangle containing $\lambda$, the Littlewood-Richardson coefficients are invariant under the following action of $\mathbb{Z}_{2} \times S_{3}$ : the non-identity element of $\mathbb{Z}_{2}$ transposes simultaneously $\mu, \nu$ and $\lambda^{\vee}$, and $S_{3}$ sorts $\mu, \nu$ and $\lambda^{\vee}(\overline{B S S})$.

The Berenstein-Zelevinsky interpretation of the Littlewood-Richardson coefficients (BZ triangles for short) (BZ) manifests all the $S_{3}$-symmetries except the commutativity. Pak and Vallejo have defined in (PV1) bijections, which are explicit linear maps, between LR tableaux, Knutson-Tao hives (KT) and BZ triangles. These bijections combined with the symmetries of BZ triangles give all the $S_{3}$ symmetries except the commutativity. The conjugation symmetry is also hidden in BZ triangles. In (GP), it is shown that it can be revealed from a bijection between web diagrams and BZ-triangles. On the other hand, the Knutson-Tao-Woodward puzzles (KTW), the most symmetrical objects, manifest only partially the conjugation symmetry through the puzzle duality, viz. $c_{\mu \nu \lambda}=c_{\nu^{t}} \mu^{t} \lambda^{t}$, since the commutativity is hidden. Interestingly, as we shall see, a similar partial conjugation symmetry, $c_{\mu \nu \lambda}=c_{\lambda^{t} \nu^{t} \mu^{t}}$, is obtained on LR tableaux through a simple bijection, denoted by $\uparrow$. In (KTW, K1, K2) bijections between hives and puzzles can be found. Recently, Purbhoo $(\overline{\mathrm{Pu}})$ introduced a new tool called mosaics, a squaretriangle-rhombus tiling model with all the rhombi arranged in the shape of a Young diagram in the corners of an hexagon. Mosaics are in bijection with puzzles and with LR tableaux, and the operation migration on mosaics, which correspond to some sequence of jeu de taquin operations on LR tableaux, reveals the hidden symmetries of puzzles. The carton rule (TY) is a recent $S_{3}$-symmetric rule but the computational complexity of the resulted visual symmetry does not seem to be improved as it is based on non trivial properties of jeu de taquin.

In (PV2), a number of Young tableau commutative symmetry maps are considered and it is shown that two of them are linear time reducible to each other and to the Schützenberger involution. (Subsequently in (DK2) and in (A3) it has been shown that the two remaining ones are identical to the others.) In this paper, we consider three Young tableau conjugation symmetry maps that appeared in (W; , HS, BSS; Z; A1, A2) and one shows that these three Young tableau conjugation symmetry maps and the commutative symmetry maps, considered in ( $\overline{\mathrm{PV} 2})$, are linear time reducible to each other and 
to the Schützenberger involution. In addition, as in the commutative case, the Young tableau conjugation symmetry maps coincide. This answers a question posed by Pak and Vallejo in (PV2).

\subsection{Summary of the results}

The conjugation symmetry map is a bijection ( $(\mathrm{PV} 2)$

$$
\varrho: L R(\lambda / \mu, \nu) \longrightarrow L R\left(\lambda^{t} / \mu^{t}, \nu^{t}\right) .
$$

Let $T$ be a tableau and $\widehat{T}$ its standardization. The Benkart-Sottile-Stroomer conjugation symmetry map $(\mathrm{BSS})$, denoted by $\varrho^{B S S}$, is the bijection

$$
\begin{array}{ccc}
\varrho^{B S S}: L R(\lambda / \mu, \nu) & \longrightarrow & L R\left(\lambda^{t} / \mu^{t}, \nu^{t}\right) \\
T & \longmapsto & \varrho^{B S S}(T)=\left[Y\left(\nu^{t}\right)\right]_{K} \cap\left[(\widehat{T})^{t}\right]_{d}
\end{array},
$$

where $\left[Y\left(\nu^{t}\right)\right]_{K}$ is the Knuth class of all tableaux with rectification the Yamanouchi tableau $Y\left(\nu^{t}\right)$ of shape the conjugate of $\nu$, and $\left[\widehat{T}^{t}\right]_{d}$ is the dual Knuth class of all tableaux of shape $\lambda^{t} / \mu^{t}$ with $Q$-symbol the transpose of $\widehat{T}$. The image of $T$ by the $B S S$-bijection is the unique tableau of shape $\lambda^{t} / \mu^{t}$ in both those two equivalence classes. Fulton showed in $(\mathrm{F})$ that the White-Hanlon-Sundaram map $\varrho^{W H S}(\mathrm{~W}$; HS) coincides with $\varrho^{B S S}$. Thus $\varrho^{B S S}(T)$ can be obtained either by tableau-switching or by the WhiteHanlon-Sundaram transformation $\varrho^{W H S}$.

Given a totally ordered finite alphabet, let $\sigma_{i}$ denote the reflection crystal operator acting on a subword over the alphabet $\{i, i+1\}$, for all $i$ (LS; Loth), and let $\sigma_{0}=\sigma_{i} \cdots \sigma_{j} \cdots \sigma_{k}$ be such that $s_{i} \cdots s_{j} \cdots s_{k}$, with $s_{l}$ the transposition $(l, l+1)$, is the longest permutation of $S_{\nu_{1}^{t}}$. The column reading word of $\varrho^{B S S}(T)$ is the Yamanouchi word of weight $\nu^{t}$ whose $Q$-symbol is the one given by the column reading word of $\widehat{T}^{t}$. The following transformation $\varrho_{3}(\mathrm{Z}$; $\mathrm{A} 1$; $\mathrm{A} 2$; $\mathrm{ACM})$ makes clear the construction of that word and affords a simple way to construct $\varrho^{B S S}(T)$

$$
\begin{aligned}
& \varrho_{3}: L R(\lambda / \mu, \nu) \quad \longrightarrow \quad L R\left(\lambda^{t} / \mu^{t}, \nu^{t}\right) \\
& T \text { with word } w \\
& \varrho_{3}(T) \text { with column word } \\
& \left(\sigma_{0} w\right)^{* \diamond}
\end{aligned}
$$

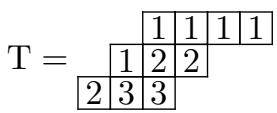

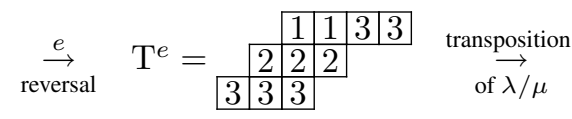

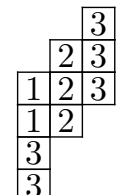

$$
\begin{aligned}
& \rightarrow \quad \begin{array}{|l|l|l|}
\cline { 2 - 3 } \multicolumn{1}{c|}{} & 1 & 1 \\
\cline { 2 - 3 } & 1 & 2 \\
\hline 1 & 2 & 3 \\
\hline 2 & 3 & \\
\hline 4 &
\end{array}=\varrho_{3}(\mathrm{~T} \\
& w=1111221332 \\
& \stackrel{\sigma_{0}}{\rightarrow} \quad \sigma_{0} w=3311222333
\end{aligned}
$$

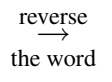

$$
\begin{aligned}
& 3332221133 \rightarrow \\
& \varrho_{3}(T)=\varrho^{B S S}(T),
\end{aligned}
$$

where $*$ denotes the dualization of a word; and $\diamond$ is the operator which transforms a Yamanouchi word of weight $\nu$, into a Yamanouchi word of weight $\nu^{t}$, by replacing the subword $i^{\nu_{i}}$ with $12 \ldots \nu_{i}$, for all $i$. The action of the operator $\diamond$ is extended to dual Yamanouchi words by putting $\left(w^{*}\right)^{\diamond}:=w^{\diamond *}$. More 
precisely, the $\diamond$ operator is a bijection between the Knuth classes of the Yamanouchi tableaux $Y(\nu)$ and $Y\left(\nu^{t}\right)$, and also between the corresponding dual Yamanouchi tableaux. The reversal $e$ of a LR tableau can be computed by the action of $\sigma_{0}$ on its word. The image of a LR or dual LR tableau $U$ under rotation of the skew-diagram by 180 degrees, with the dualization $*$ of its word is denoted by $U^{\bullet}$; and the image of $U$ under the rotation and transposition of the skew-diagram, with the action of the operation $\diamond$ on its word is denoted by $U$. Again $\bullet$ and $\bullet$ are involution maps. Then

$$
\begin{gathered}
\varrho_{3}(T)=T^{e \bullet}=T^{\bullet \bullet e}=T^{\bullet \diamond} e \text { and } \\
\left(\sigma_{0} w\right)^{* \diamond}=\left(\sigma_{0} w\right)^{\diamond *}=\sigma_{0}\left(w^{\diamond *}\right) \text { is the column word of } T^{e \bullet}=\left[Y\left(\nu^{t}\right)\right]_{K} \cap\left[(\widehat{T})^{t}\right]_{d} .
\end{gathered}
$$

In the two next sections we shall develop the necessary machinery to show the above identities. Bijection ๑ appeared originally in (Z) with a different formulation. In (A1; $\mathrm{A} 2)$ the bijection $e$, defined differently and based on a modified insertion, is composed with the last one to give $\rho_{3}$. Here we stress the composition of $e \bullet$ with $\diamond$.

Following the ideas introduced in (PV2), we address, in Section 4, the problem of studying the computational cost of the conjugation symmetry map $\varrho^{B S S}$ utilizing what is known as Relative Complexity, an approach based on reduction of combinatorial problems. To this aim we use the version $\varrho_{3}$. We consider only linear time reductions; since the bijections we consider require subquadratic time the reductions have to preserve that. Let $\mathcal{A}$ and $\mathcal{B}$ be two possibly infinite sets of finite integer arrays, and let $\delta: \mathcal{A} \longrightarrow \mathcal{B}$ be an explicit map between them. We say that $\delta$ has linear cost if $\delta$ computes $\delta(A) \in \mathcal{B}$ in linear time $O(\langle A\rangle)$ for all $A \in \mathcal{A}$, where $\langle A\rangle$ is the bit-size of $A$. The transposition of the recording matrix of a LR tableau is the recording matrix of a tableau of normal shape. We have then a linear map $\tau$ which defines a bijection between tableaux of normal shape and LR tableaux (Lee1; Lee2; PV2; O). As the rotation map $\bullet$ and $\tau$ are linear maps, so maps of linear cost, the reversal $T^{e}$ of a LR tableau $T$ can be linearly reduced to the evacuation $E$ of the corresponding tableau $\tau(T)=P$ of normal shape, i.e. $\tau\left(P^{E}\right)=T^{e}$. Additionally, in Algorithm 4.1, it is proved that the bijection $\downarrow$, exhibiting the symmetry $c_{\mu \nu \lambda}=c_{\lambda^{t} \nu^{t} \mu^{t}}$, is of linear cost. The following commutative scheme shows that the conjugation symmetry map $\varrho_{3}$, and therefore $\varrho^{B S S}$ and $\varrho^{W H S}$, is linear equivalent to the Schützenberger involution or evacuation map on tableaux of normal shape,

Theorem 1.1 The following commutative scheme holds

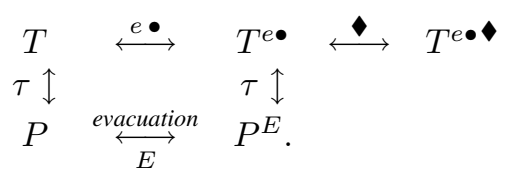

Theorem 1.2 The conjugation symmetry maps $\varrho^{B S S}$, $\varrho^{W H S}$ and $\varrho_{3}$ are identical, and linear time equivalent with the Schützenberger involution $E$ and with the reversal map $e$.

We may now extend the list of linear equivalent Young tableau maps established in (PV2), Section 2, Theorem 1.

Theorem 1.3 (PV2) The following maps are linearly equivalent:

(1) RSK correspondence.

(2) Jeu de taquin map. 
(3) Littlewood-Robinson map.

(4) Tableau switching map s.

(5) Evacuation (Schützenberger involution) E for normal shapes.

(6) Reversal e.

(7) First fundamental symmetry map.

(8) Second fundamental symmetry map.

Corollary 1.1 The following maps are linearly equivalent:

(1) RSK correspondence.

(2) Jeu de taquin map.

(3) Littlewood-Robinson map.

(4) Tableau switching map s.

(5) Evacuation (Schützenberger involution) E for normal shapes.

(6) Reversal e.

(7) First fundamental symmetry map.

(8) Second fundamental symmetry map.

(9) Third fundamental symmetry map.

(10) $\varrho^{W H S}$ conjugation symmetry map.

(11) $\varrho^{B S S}$ conjugation symmetry map.

(12) $\varrho_{3}$ conjugation symmetry map.

In particular, first and second fundamental symmetry maps are identical (DK2); first and third fundamental symmetry maps are identical (A3); $\varrho^{W H S}$ and $\varrho^{B S S}$ are identical conjugation symmetry maps $(\bar{F})$, and the same happens with $\varrho^{B S S}$ and $\varrho_{3}$.

\section{Preliminaries}

\subsection{Young diagrams and transformations}

A partition (or normal shape) $\lambda$ is a sequence of non-negative integers $\lambda=\left(\lambda_{1}, \lambda_{2}, \ldots, \lambda_{\ell}\right)$, with $\lambda_{1} \geq$ $\lambda_{2} \geq \cdots \geq \lambda_{\ell} \geq 0$. The number of parts is $\ell(\lambda)=\ell$ and the weight is $|\lambda|=\lambda_{1}+\lambda_{2}+\cdots+\lambda_{\ell}$. (For convenience we allow zero parts.) The Young diagram of $\lambda$ is the collection of boxes $\left\{(i, j) \in \mathbb{Z}^{2} \mid 1 \leq\right.$ $\left.i \leq \ell, 1 \leq j \leq \lambda_{i}\right\}$. The English convention is adopted in drawing such a diagram. Throughout the paper we do not make distinction between a partition $\lambda$ and its Young diagram $(\mathrm{P})$. Given partitions $\lambda$, $\mu$, we say that $\mu \subseteq \lambda$ if $\mu_{i} \leq \lambda_{i}$ for all $i>0$. If $\left(r^{\ell}\right)$ is a $r \times \ell$ rectangle containing $\lambda$, the complement of $\lambda$ regarding that rectangle is the partition $\lambda^{\vee}=\left(r-\lambda_{\ell}, \ldots, r-\lambda_{1}\right)$. We define $\lambda^{t}$ the conjugation or transposition of $\lambda$ as the image of $\lambda$ under the transposition $(i, j) \rightarrow(j, i)$. For example, let $r=4$ and $\ell=3$. The Young diagram of $\lambda=(3,2,2)$ and its transpose $\lambda^{t}=(3,3,1)$ are depicted below; and $\lambda^{\vee}=(2,2,1)$, $\left(\lambda^{t}\right)^{\vee}=\left(\lambda^{\vee}\right)^{t}=(3,2,0)$ are depicted by dotted boxes
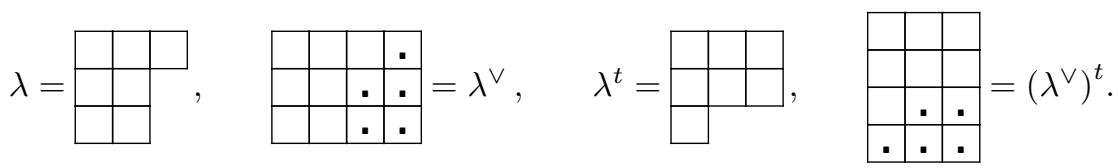

A skew-diagram (skew-shape) $\lambda / \mu$ is $\left\{(i, j) \in \mathbb{Z}^{2} \mid(i, j) \in \lambda,(i, j) \notin \mu\right\}$ the collection of boxes in $\lambda$ which are not in $\mu$. When $\mu$ is the null partition, the skew-diagram $\lambda / \mu$ equals the Young diagram $\lambda$. The 
number of boxes in $\lambda / \mu$ is $|\lambda / \mu|=|\lambda|-|\mu|$. The transpose (conjugate shape) $(\lambda / \mu)^{t}$ is the skew-diagram $\lambda^{t} / \mu^{t}$ obtained by transposing the skew-diagram $\lambda / \mu$. Let $r=\lambda_{1}$. The rotation (dual shape) $(\lambda / \mu)^{*}$ is the image of $\lambda / \mu$ by rotation of 180 degrees, or the image of $\lambda / \mu$ under $(i, j) \longrightarrow(\ell-i+1, r-j+1)$. Equivalently $(\lambda / \mu)^{*}=\mu^{\vee} / \lambda^{\vee}$. In particular, $\lambda^{*}$ is the skew-diagram $r^{\ell} / \lambda^{\vee}$. The dual conjugate shape $(\lambda / \mu)^{\diamond}$ is the image of $\lambda / \mu$ under $(i, j) \longrightarrow(r-j+1, \ell-i+1)$. The map $\diamond$ is the composition of the transposition with the rotation maps $\diamond=* t=t *$. In particular, $\lambda^{\diamond}=\ell^{r} /\left(\lambda^{\vee}\right)^{t}$. For instance, if $\mu=(2) \subset \lambda=(4,3,1)$, we have

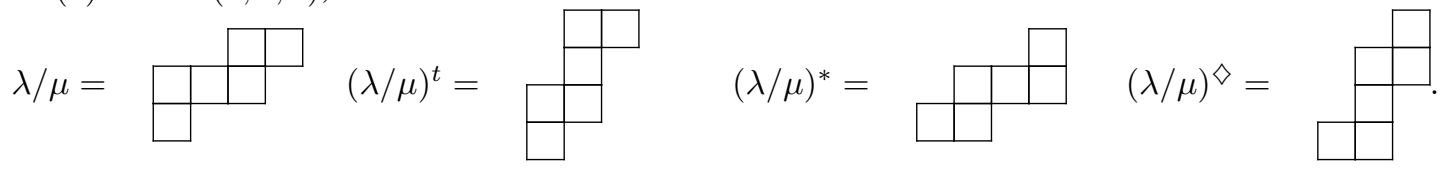

\subsection{Tableaux and words}

The Littlewood-Richardson (LR for short) numbering (reading) of the boxes of a skew-diagram $\lambda / \mu$ is an assignment of the labels $1,2, \ldots$ which sorts the boxes of $\lambda / \mu$ in increasing order from right to left along each row, starting in the top row and moving downwards; and the column LR numbering of the boxes sorts in increasing order, from right to left along each column, starting in the rightmost column and moving downwards. Analogously the reverse LR numbering and the column LR numbering of $\lambda / \mu$ are defined.

Example 2.1 If $\lambda / \mu=\sqrt{\square_{1}}{ }_{\square}{ }_{\square}$, the LR-numbering, column LR-numbering and the corresponding re-

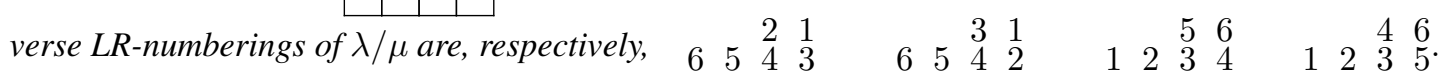

Clearly, the column LR-numbering of $\lambda / \mu$ is the LR-numbering of $(\lambda / \mu)^{\diamond}$, and the reverses of LRnumbering and column LR-numbering of $\lambda / \mu$ are, respectively, the LR-numbering of $(\lambda / \mu)^{*}$ and $(\lambda / \mu)^{t}$.

A Young tableau $T$ of shape $\lambda / \mu$ is a filling of the boxes of the skew-diagram $\lambda / \mu$ with positive integers in $\{1, \ldots, t\}$ which is increasing in columns from top to bottom and non-decreasing in rows from left to right. When $\mu$ is the empty partition we say that $T$ has normal shape $\lambda$. The word $w(T)$ of a Young tableau $T$ is the sequence obtained by reading the entries of $T$ according to its LR numbering, that is, reading right-to-left the rows of $T$, from top to bottom. The column word $w_{c o l}(T)$ is the word obtained according the column LR numbering. The weight of $T$ is the weight of of its word. Denote by $Y T(\lambda / \mu, m)$ the set of Young tableaux of shape $\lambda / \mu$ and weight $m=\left(m_{1}, \ldots, m_{t}\right)$.

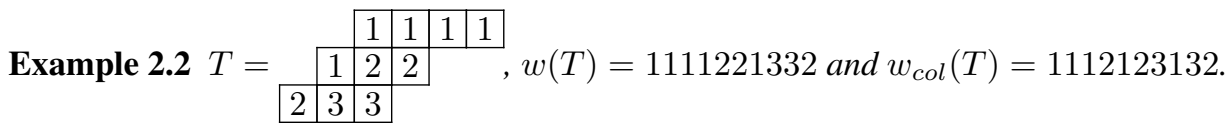

A Young tableau with $s$ boxes is standard if it is filled with $\{1, \ldots, s\}$ without repetitions. Given a tableau $T$ of weight $m$, the standardization of $T$, denoted by $\widehat{T}$, is obtained by replacing, west to east, the letters 1 in $\mathrm{T}$ with $1,2, \ldots, m_{1}$; the letters 2 with $m_{1}+1, \ldots, m_{1}+m_{2}$; and so on. The standardization $\widehat{w}$ of a word $w$ is defined accordingly, from right to left. For instance, the standardization of the tableau $T$ in the

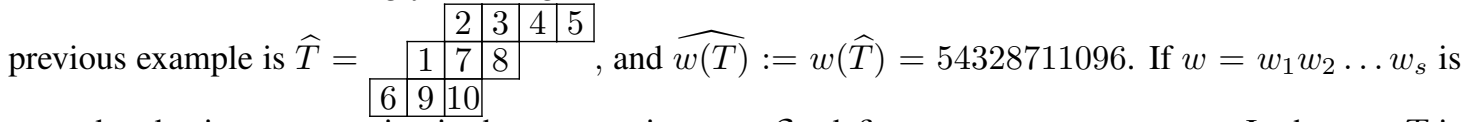
a word and $\alpha$ is a permutation in the symmetric group $\mathcal{S}_{s}$, define $\alpha w=w_{\alpha(1)} \ldots w_{\alpha(s)}$. In the case $T$ is standard we have $w_{\text {col }}(\widehat{T})=\operatorname{rev} w\left(\widehat{T}^{t}\right)$, with rev the reverse permutation. 
A Young tableau $T$ is said a Littlewood-Richardson (LR for short) tableau if its word, when read from the beginning to any letter, contains at least as many letters $i$ as letters $i+1$, for all $i$. More generally, a word such that every prefix satisfies this property is called a lattice permutation or a Yamanouchi word. Notice that the column word of a LR-tableau is also a Yamanouchi word of the same weight. Denote by $L R(\lambda / \mu, \nu)$ the set of LR tableaux of shape $\lambda / \mu$ and weight $\nu$. When $\mu=0$ we get the Yamanouchi tableau $Y(\nu)$, the unique tableau of shape and weight $\nu$. In Example 2.2, $T$ is a LR tableau with Yamanouchi word $w(T)=1111221332$ and column word $w_{\text {col }}(T)=1112123132$.

There is an one-to-one correspondence between Yamanouchi words of weight $\nu$ and standard tableaux of shape $\nu$. Let $w=w_{1} w_{2} \cdots w_{s}$ be a Yamanouchi word and put the number $k$ in the $w_{k}$ th row of the diagram $\nu$. The labels of the $i$ th row are the $k$ 's such that $w_{k}=i$, thus the length is $\nu_{i}$ and the shape is $\nu$. We denote this standard tableau by $U(w)$. In Example 2.2 $w=1111221332$, and $U(w)=$\begin{tabular}{|l|l|l|l|l|}
\hline 1 & 2 & 3 & 4 & 7 \\
\hline 5 & 6 & 10 & &
\end{tabular} where the entries of the $i$ th row are the positions of the $i$ 's in the LR reading of $T$.

\subsection{Matrices and tableaux}

Given $T \in Y(\lambda / \mu, m)$, let $M=\left(M_{i j}\right)_{1 \leq i \leq \ell(\lambda), 1 \leq j \leq t}$ be a matrix with non-negative entries such that $M_{i j}$ is the number of $j^{\prime} s$ in the $i$ th row of $T$, called the recording matrix of $T$ (Lee1; Lee2; PV2). The recording matrix of a tableau of normal shape is an upper triangular matrix, and the recording matrix of an LR tableau is a lower triangular matrix. Thus we have an one-to-one correspondence between LR tableaux and tableaux of normal shape as follows. Considering $T$ in Example 2.2, the recording matrix of $T$ is $M=\left(\begin{array}{lll}4 & 0 & 0 \\ 1 & 2 & 0 \\ 0 & 1 & 2\end{array}\right)$. On the other hand, the transposition $M^{t}=\left(\begin{array}{lll}4 & 1 & 0 \\ 0 & 2 & 1 \\ 0 & 0 & 2\end{array}\right)$ encodes the tableau $B=$\begin{tabular}{l|l|l|l|l|}
\hline & 1 & 1 & 1 & 2 \\
\hline 2 & 2 & 3 & &
\end{tabular} of normal shape $\nu$ and weight $\lambda-\mu$. For two Young diagrams $\mu$ and $\nu$, define $\nu \circ \mu=\left(\nu_{1}+\mu_{1}, \ldots, \nu_{1}+\mu_{\ell}, \nu_{1}, \ldots, \nu_{r}\right) /\left(\mu_{1}+\nu_{1}, \ldots, \mu_{\ell}+\nu_{1}\right), \ell=\ell(\mu), r=\ell(\nu)$. Then with $\mu=(1), B \circ Y(\mu)=$\begin{tabular}{ll|l|l|l|l|l}
\hline & 1 & 1 & 1 & 2 \\
\hline 2 & 2 & 3 &
\end{tabular}$\in L R(\nu \circ \mu, \lambda)$. Given partitions $\lambda, \mu, \nu$ such that $|\lambda|=|\mu|+|\nu|$, define $C F(\nu, \mu, \lambda)=\{B \in Y T(\nu, \lambda-\mu): B \circ Y(\mu) \in \operatorname{LR}(\nu \circ \mu, \lambda)\}$ (PV2). The map $\tau: L R(\lambda / \mu, \nu) \rightarrow C F(\nu, \mu, \lambda)$ such that $\tau(M)$ is the tableau of normal shape with recording matrix $M^{t}$, where $M$ is the recording matrix of $T$, is a bijection. Taking again Example 2.2, we have $\tau(T)=B$.

\subsection{Rotation and transposition of $L R$ tableaux}

Given an integer $i$ in $\{1, \ldots, t\}$, let $i^{*}:=t-i+1$. Given a word $w=w_{1} w_{2} \cdots w_{s}$, over the alphabet $\{1, \ldots, t\}$, of weight $m=\left(m_{1}, \ldots, m_{t}\right), w^{*}:=w_{s}^{*} \cdots w_{2}^{*} w_{1}^{*}$ is the dual word of $w$ and $m^{*}=\left(m_{t}, \ldots, m_{1}\right)$ its weight. Indeed $w^{* *}=w$. A dual Yamanouchi word is a word whose dual word is Yamanouchi. Given a Young tableau $\mathrm{T}$ of shape $\lambda / \mu$ and weight $\left(m_{1}, \ldots, m_{t}\right), \mathrm{T}^{\bullet}$ denotes the Young tableau of shape $(\lambda / \mu)^{*}$ and weight $m^{*}$, obtained from T by replacing each entry $i$ with $i^{*}$, and then rotating the result by 180 degrees. The word of $\mathrm{T}^{\bullet}$ is $w(\mathrm{~T})^{*}$, and $\mathrm{T}^{\bullet \bullet}=\mathrm{T}$. A dual LR tableau is a tableau whose word is a dual Yamanouchi word. $\operatorname{LR}\left(\lambda / \mu, \nu^{*}\right)$ denotes the set of dual LR tableaux of shape $\lambda / \mu$ and weight $\nu^{*}$, and is the image of $\operatorname{LR}\left((\lambda / \mu)^{*}, \nu\right)$ under the rotation map $\bullet$. Thus the rotation 
map • defines a bijection between $\operatorname{LR}\left((\lambda / \mu)^{*}, \nu^{*}\right)$ and $\operatorname{LR}(\lambda / \mu, \nu)$. Given a Yamanouchi word $w$ of weight $\nu$, define the standard tableau $U\left(w^{*}\right)$ of shape $\nu^{*}$ such that the label $k$ is in row $i$ if and only if $w_{s-i+1}=k^{*}$. Thus $U\left(w^{*}\right)=U(w)^{\bullet}$ and this affords a bijection between dual Yamanouchi words of weight $\nu^{*}$ and standard tableaux of shape $\nu^{*}$. The rotation map $\bullet$ is a linear map: $M=\left(M_{i j}\right)$ is the recording matrix of $T$ if and only if the recording matrix of $T^{\bullet}$ is $\left(M_{s+1-i, t-j+1}\right)$.

There is another natural bijection, denoted by $\downarrow$, between LR tableaux of conjugate weight and dual conjugate shape, see (Z, A1, A2). Given a Yamanouchi word $w$ of weight $\nu=\left(\nu_{1}, \ldots, \nu_{t}\right)$, write $\nu^{t}=\left(\nu_{1}^{t}, \ldots, \nu_{k}^{t}\right)$ and observe that $w$ is a shuffle of the words $12 \ldots \nu_{i}^{t}$ for all $i$, and its dual word is a shuffle of the words $t t-1 \cdots t-\nu_{i}^{t}+1$, for all $i$. Thus, we define $w^{\diamond}$ as the Yamanouchi word of weight $\nu^{t}$ obtained by replacing the subword consisting only on the letters $i$ with the subword $12 \cdots \nu_{i}$, for each $i$. The operation $\diamond$ is defined on dual Yamanouchi words by $w^{* \diamond}:=w^{\diamond *}=$, giving rise to a dual Yamanouchi word of weight $\nu^{* t}$. The word $w^{\diamond *}$ can be obtained in just only one step: replace the subword of $w$ consisting only on the letters $i$ with the subword $\nu_{1} \nu_{1}-1 \cdots \nu_{1}-\nu_{i}+1$, for all $i$. Clearly, $U\left(w^{\diamond}\right)=U(w)^{t}$ is of shape $\nu^{t}$, and $U\left(w^{* \diamond}\right)=U(w)^{\bullet t}$ is of shape $\nu^{*}$. Given $\mathrm{T} \in \operatorname{LR}(\lambda / \mu, \nu)$ $\left(\operatorname{LR}\left(\lambda / \mu, \nu^{*}\right)\right)$ with word $w$, define $\mathrm{T}^{\star}$ as the LR tableau of shape $(\lambda / \mu)^{\diamond}$ and weight $\nu^{t}$ obtained from $\mathrm{T}$ by replacing the word $w$ with $w^{\diamond}$, and then rotating the result by 180 degrees and transposing. Then $\checkmark: \operatorname{LR}(\lambda / \mu, \nu)\left(\operatorname{LR}\left(\lambda / \mu, \nu^{*}\right)\right) \longrightarrow \operatorname{LR}\left((\lambda / \mu)^{\diamond}, \nu^{t}\right) \operatorname{LR}\left((\lambda / \mu)^{\diamond}, \nu^{* t}\right)$ is a bijection such that $T^{\diamond}$ has column word $w^{\diamond}$ and $\mathrm{T}^{\bullet}=\mathrm{T}$. Since $\bullet=\bullet \bullet, T^{\bullet}=T^{\bullet \bullet} \in \operatorname{LR}\left((\lambda / \mu)^{t}, \nu^{t *}\right)\left(\operatorname{LR}\left((\lambda / \mu)^{t}, \nu^{t}\right)\right)$ has column word $w^{* \diamond}$.

Example 2.3 $\mathrm{T}=$\begin{tabular}{|l|l|l|l|}
\cline { 2 - 2 } & 1 & 1 \\
\hline & 1 & 2 & 2
\end{tabular} is a LR tableau with word $w=1122131$ of weight $\nu=(4,2,1)$. Then

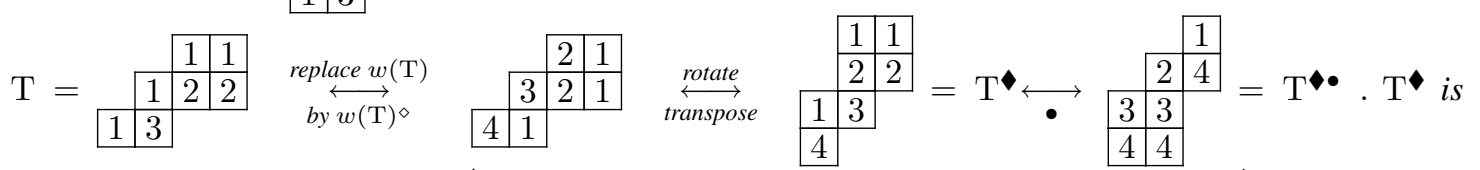
a LR tableau with shape $(\lambda / \mu)^{\diamond}$ and column word $w^{\diamond}=1212314$ of weight $\nu^{t} . \mathrm{T}^{\bullet \bullet}$ is a dual LR tableau with shape $(\lambda / \mu)^{t}$ and column word $w^{\diamond *}=1423434$ of weight $\nu^{t *}$, where $U(w)=$\begin{tabular}{|l|l|l|l|}
\hline & 2 & 5 & 7 \\
\hline 3 & 4 & \\
\hline 6 &
\end{tabular},

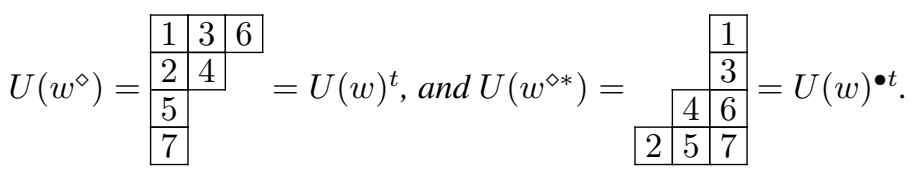

\section{Conjugation symmetry maps}

\subsection{Knuth equivalence and dual Knuth equivalence}

Whenever partitions $\nu \subset \mu \subset \lambda$, we say that $\lambda / \mu$ extends $\mu / \nu$. An inside corner of $\lambda / \mu$ is a box in the diagram $\mu$ such that the boxes below and to the right are not in $\mu$. When a box extends $\lambda / \mu$, this box is called an outside corner. Let $\mathrm{T}$ be a Young tableau and let $b$ be an inside corner for $T$. A contracting slide (Sch; BSS) of $T$ into the box $b$ is performed by moving the empty box at $b$ through $\mathrm{T}$, successively interchanging it with the neighboring integers to the south and east according to the following rules: $(i)$ if the empty box has only one neighbor, interchange with that neighbor; $(i i)$ if it has two unequal neighbors, interchange with the smaller one; and (iii) if it has two equal neighbors, interchange with that one to the 
south. The empty box moves in this fashion until it has become an outside corner. This contracting slide can be reversed by performing an analogous procedure over the outside corner, called an expanding slide. Performing a contracting slide over each inside corner of $T$ reduces $T$ to a tableau $T^{\mathrm{n}}$ of normal shape. This procedure is known as jeu de taquin. $T^{\mathrm{n}}$ is independent of the particular sequence of inside corners used (Th), and so $\mathrm{T}^{\mathrm{n}}$ is called the rectification of T. A word $w$ corresponds by RSK-correspondence to a pair $(P(w), Q(w))$ of tableaux of the same shape, with $Q(w)$ standard, called the $Q$-symbol or recording tableau of $w$. Here we consider a variation of RSK-correspondence known as the Burge correspondence (B; F). Given $w=w_{1} w_{2} \cdots w_{s}, P(w)$ is the insertion tableau obtained by column insertion of the letters of $w$ from left to right $(\mathrm{F})$. The corresponding recording tableau $Q(w)$ is obtained by placing in $1,2, \ldots, s$. If $w$ is the word of $\mathrm{T}$ then $P(w)=T^{\mathrm{n}}$. Insertion can be translated into the language of Knuth elementary transformations. Two words $w$ and $v$ are said Knuth equivalent if they have the same insertion tableau. Each Knuth class is in bijection with the set of standard tableaux with shape equal to the unique tableau in that class. Two tableaux $T$ and $R$ are Knuth equivalent, written $T \equiv R$, if and only if $P(w(T))=P(w(R))$. Equivalently, $T^{\mathrm{n}}=R^{\mathrm{n}}$, i.e. one of them can be transformed into the other one by a sequence of jeu de taquin slides. The insertion tableau of a Yamanouchi word $w$ with partition weight $\nu$, is the Yamanouchi tableau $Y(\nu)$. The recording tableau of a Yamanouchi word $w$ is $U(w)$.

Two tableaux $T$ and $R$ of the same shape are dual equivalent, written $T \stackrel{d}{\equiv} R$, if any sequence of contracting slides and expanding slides that can be applied to one of them, can also be applied to the other, and the sequence of shape changes is the same for both $(\mathrm{H}, \mathrm{F})$. Dual equivalence may also be characterized by recording tableaux: $T \stackrel{d}{\equiv} R$ if and only if $Q(w(T))=Q(w(R))$. Thus two tableaux of the same normal shape are dual equivalent. Let $S$ and $T$ be tableaux such that $\mathrm{T}$ extends $\mathrm{S}$, and consider the set union $\mathrm{S} \cup \mathrm{T}$. The tableau switching (BSS) is a procedure based on jeu de taquin elementary moves on two alphabets that transforms $\mathrm{S} \cup \mathrm{T}$ into $\mathrm{A} \cup \mathrm{B}$, where $B$ is a tableau Knuth equivalent to $T$ which extends $\mathrm{A}$, and $A$ is a tableau Knuth equivalent to $S$. We write $\mathrm{S} \cup \mathrm{T} \stackrel{\mathrm{s}}{\longrightarrow} \mathrm{A} \cup \mathrm{B}$. In particular, if $S$ is of normal shape, $\mathrm{A}=\mathrm{T}^{\mathrm{n}}$, and $S=B^{\mathrm{n}}$. Switching of $S$ with $T$ may be described as follows: $\widehat{T}$ is a set of instructions telling where expanding slides can be applied to $S$. Thus switching and dual equivalence are related as below and tableaux are completely characterized by dual and Knuth equivalence.

Theorem $3.1(\underline{\mathrm{H}})$ Let $T$ and $U$ be tableaux with the same normal shape and let $W$ be a tableau which extends $T$. (1) If $T \cup W \stackrel{s}{\longrightarrow} Z \cup X$ and $U \cup W \stackrel{s}{\longrightarrow} Z \cup Y$, then $X \stackrel{d}{\equiv} Y$.

(2) Let $\mathcal{D}$ be a dual equivalence class and $\mathcal{K}$ be a Knuth equivalence class, both corresponding to the same normal shape. Then, there is a unique tableau in $\mathcal{D} \cap \mathcal{K}$.

Algorithm to construct $\mathcal{D} \cap \mathcal{K}$ : Let $U \in \mathcal{D}$ and let $V \in \mathcal{K}$ be the only tableau with normal shape

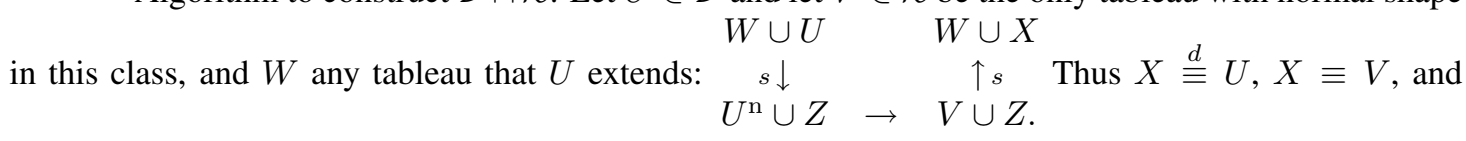
$\mathcal{D} \cap \mathcal{K}=\{X\}$. since two words in the same Knuth class can not have the same $Q$-symbol.

\subsection{The transposition of the rotated reversal $L R$ tableau}

Given a tableau $\mathrm{T}$ of normal shape, the evacuation $\mathrm{T}^{E}$ is the rectification of $\mathrm{T}^{\bullet}$, that is, $\mathrm{T}^{\mathrm{E}}=\mathrm{T}^{\bullet \mathrm{n}}$. $\mathrm{T}^{E}$ is also obtained either as the insertion tableau of the word $w(\mathrm{~T})^{*}$; or according to the Schützenberger evacuation algorithm; or applying the reverse jeu de taquin slides to $T$, in the smallest rectangle containing $T$, to obtain $T^{\mathrm{a}}$ the anti-normal form $T$. Thus $T^{\bullet}=T^{E}=T^{\bullet n}$. If $w$ is a Yamanouchi word, by duality 
of Burge correspondence, $Q\left(w^{*}\right)=U(w)^{E}=U(w)^{\bullet n}=U(w)^{\bullet} \cdot$. Given $w \equiv \mathrm{Y}(\nu)$, we may now define the word $w^{\diamond}$ as being the unique word satisfying $w^{\diamond} \equiv \mathrm{Y}\left(\nu^{t}\right)$ such that $Q\left(w^{\diamond}\right)=Q(w)^{T}=U(w)^{t}$. Since $\left(w^{\diamond}\right)^{\diamond}=w$, the map $w \mapsto w^{\diamond}$ establishes a bijection between the Knuth classes of $Y(\nu)$ and $Y\left(\nu^{t}\right)$. The word $w^{*}$ is the unique word satisfying $w \equiv \mathrm{Y}\left(\nu^{*}\right)$ such that $Q\left(w^{*}\right)=U(w)^{\bullet n}$, and $w^{\diamond *}$ is the unique word satisfying $w^{\diamond *} \equiv \mathrm{Y}\left(\nu^{t *}\right)$ such that $Q\left(w^{\diamond *}\right)=U(w)^{E t}$

Given a tableau $T$ of any shape, the reversal $T^{e}$ is the unique tableau Knuth equivalent to $\mathrm{T}^{\bullet}$, and dual equivalent to $\mathrm{T}(\overline{\mathrm{BSS}})$. By Theorem 3.1, $T^{e}=\left[T^{\mathrm{n} \mathrm{E}}\right]_{K} \cap[T]_{d}$, where []$_{K}$ denotes Knuth class and [ ] $d_{d}$ dual Knuth class. If $T$ has normal shape, $T^{E}=T^{e}$. If $T \in \operatorname{LR}(\lambda / \mu, \nu)$, then $T^{e}$ is the only tableau Knuth equivalent to $Y\left(\nu^{*}\right)$ and dual equivalent to $T$. Since crystal reflection operators, for the definition see (LS; Loth), preserve the $Q$-symbol, we may in the case of LR tableaux characterize explicitly the word of $T^{e}$ as follows. Let $w$ be a Yamanouchi word of weight $\nu=\left(\nu_{1}, \ldots, \nu_{t}\right)$, and let $\sigma_{i}$ denote the reflection crystal operator acting on the subword over the alphabet $\{i, i+1\}$, for all $i$. If $s_{i_{r}} \cdots s_{i_{1}}$ is the longest permutation in $\mathcal{S}_{t}$, put $\sigma_{0}:=\sigma_{i_{r}} \cdots \sigma_{i_{1}}$. Then $\sigma_{0} w$ is a dual Yamanouchi word of weight $\nu^{*}$. Moreover, $w \equiv w^{\prime}$ if and only if $\sigma_{i}(w) \equiv \sigma_{i}\left(w^{\prime}\right)$, and $Q(w)=Q\left(\sigma_{i}(w)\right)$. Thus, we have proven the following

Theorem 3.2 Let $\mathrm{T}$ be a LR tableau with shape $\lambda / \mu$ and word $w$. Then $\mathrm{T}^{e}$ is the dual LR tableau of shape $\lambda / \mu$ and word $\sigma_{0} w$, and $T^{e}$ is the LR tableau of shape $(\lambda / \mu)^{t}$ and column word $\left(\sigma_{0} w\right)^{\diamond *}$.

Corollary 3.1 $T^{e}$ is the unique tableau Knuth equivalent to $Y\left(\nu^{t}\right)$ and dual equivalent to $\widehat{T}^{t}$.

Proof: It is enough to see that the column words of $T^{e} \bullet$ and $\widehat{T}^{t}$ have the same $Q-$ symbol. Let $\widehat{w}$ be the word of $\widehat{T}$. As rev $\widehat{w}$, the reverse word of $\widehat{T}$, is the column word of $\widehat{T}^{t}$, then $Q(\operatorname{rev} \widehat{w})=Q(\widehat{w})^{E t}=$ $Q(w)^{E t}=Q\left(w^{\diamond *}\right)=Q\left(\sigma_{0}\left(w^{\diamond *}\right)\right)=Q\left(\left(\sigma_{0} w\right)^{\diamond *}\right)$.

We recall that the action of crystal reflection operators on words corresponds to jeu de taquin slides on two-row tableaux. In particular, if $w$ is a Yamanouchi word of weight $\nu$ and $\theta_{i}$ denotes the jeu de taquin action on the consecutive rows $i$ and $i+1$ of $U(w)$, then $\theta_{i} U(w)$ is a tableau of skewshape $(i i+1) \nu$ such that any two consecutive rows define a two-row tableau of normal or anti-normal shape. The labels of the $j$-th row of $\theta_{i} U(w)$ are precisely the $k$ 's such that $\left(\sigma_{i} w\right)_{k}=j$. Put $\theta_{0}:=$ $\theta_{i_{r}} \ldots \theta_{i_{1}}$ with $i_{r}, \ldots, i_{1}$ as in $\sigma_{0}$. Thus $\theta_{0} U(w)=U(w)^{\mathrm{a}}$ and $Q\left(\sigma_{0} w\right)=U(w)^{\mathrm{a}}$. This defines the

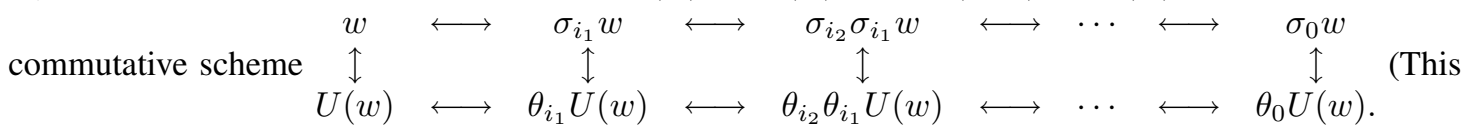
was the procedure in (A1).) Similarly, if $\sigma_{i} T$ denotes the tableau obtained by the action of $\sigma_{i}$ on its word,

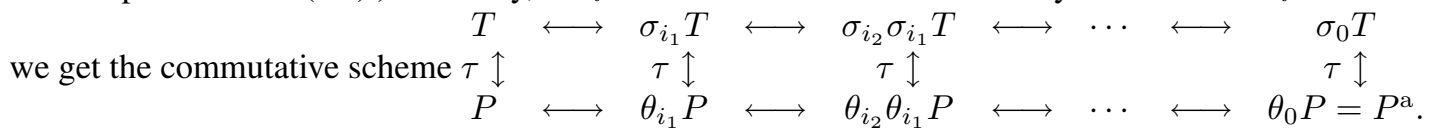

Theorem 3.3 Let $T$ be a LR tableau and $\tau(T)=P$. Then, the following commutative scheme holds

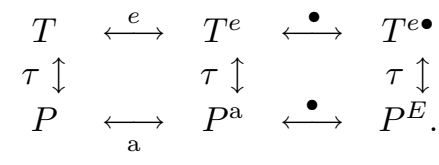




\subsection{Main bijections}

As already mentioned bijections $\varrho^{W H S}$ and $\varrho^{B S S}$ are identical. Let

$$
\begin{array}{cccc}
\varrho^{B S S}: \quad L R(\lambda / \mu, \nu) & \rightarrow & L R\left(\lambda^{t} / \mu^{t}, \nu^{t}\right) & \\
T & \mapsto & \varrho^{B S S}(T)=\left[Y\left(\nu^{t}\right)\right]_{K} \cap\left[\widehat{T}^{t}\right]_{d} &
\end{array}
$$

The image of $T$ by the $B S S$-bijection is the unique tableau of shape $\lambda^{t} / \mu^{t}$ whose rectification is $Y\left(\nu^{t}\right)$ and the $Q$-symbol of the column reading word is $Q(T)^{E t}$. The idea behind this bijection can be told as follows: $\widehat{T}$ constitutes a set of instructions telling where expanding slides can be applied to $Y(\mu)$. Then $\widehat{T}^{t}$ is a set of instructions telling where expanding slides can be applied to $Y(\mu)^{t}$. Tableau-switching provides an algorithm to give way to those instructions:

$$
\begin{aligned}
& Y(\mu) \cup T \underset{\text { of T }}{\stackrel{\text { standardization }}{\longrightarrow}} Y(\mu) \cup \widehat{T} \underset{\text { of } \widehat{T}}{\stackrel{\text { transposition }}{\longrightarrow}} Y\left(\mu^{t}\right) \cup \widehat{T}^{t} \quad Y\left(\mu^{t}\right) \cup \varrho^{B S S}(T) \\
& \begin{array}{cc}
\stackrel{\downarrow}{\downarrow s} & \uparrow s \\
\left(\widehat{T}^{t}\right)^{\mathrm{n}} \cup Z & \mapsto \quad Y\left(\nu^{t}\right) \cup Z
\end{array}
\end{aligned}
$$

Then $\varrho^{B S S}(T) \equiv Y\left(\nu^{t}\right)$ and $\varrho^{B S S}(T) \equiv{ }_{d} \widehat{T}^{t}$.

Example 3.4 Let $T$ in $L R(\lambda / \mu, \nu)$ with $\mu=(2,1), \nu=(5,3,2)$ and $\lambda=(6,4,3)$ :

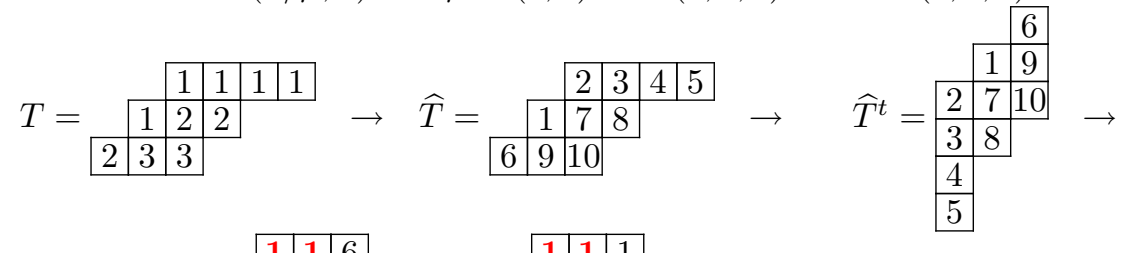

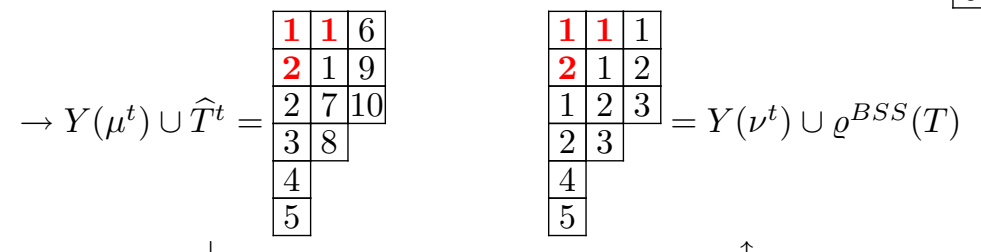

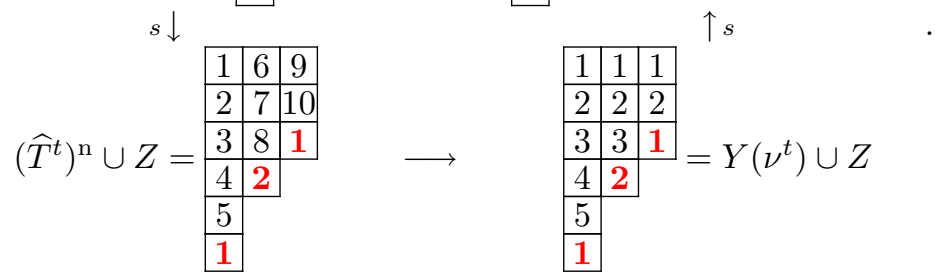

Let

$$
\begin{array}{cccc}
\varrho_{3}: \quad L R(\lambda / \mu, \nu) & \rightarrow & L R\left(\lambda^{t} / \mu^{t}, \nu^{t}\right) & \\
T & \mapsto & \varrho_{3}(T)=T^{e} \bullet \\
w & \mapsto & \sigma_{0} w^{* \diamond} & \text { (Z; } ; \text { A1; }, \text { A2 }) .
\end{array}
$$

As $T^{e \bullet}$ is the unique tableau Knuth equivalent to $Y\left(\nu^{t}\right)$ and dual equivalent to $(\widehat{T})^{t}$, we have Corollary $3.2 \varrho^{B S S}$ and $\varrho_{3}$ are identical bijections. 
Example 3.5 Let $T$ in $L R(\lambda / \mu, \nu)$ as before:

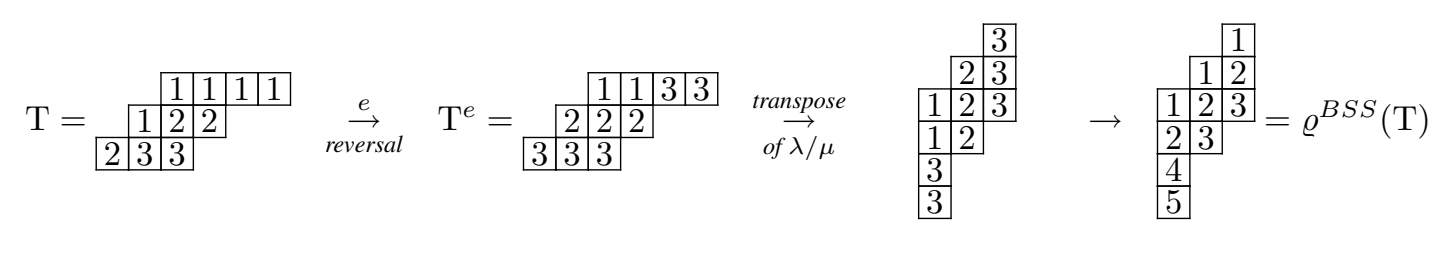

$$
\begin{aligned}
& w=1111221332 \quad \rightarrow \quad \sigma_{0} w=3311222333 \stackrel{\text { reverse }}{\longrightarrow} 3332221133 \rightarrow \quad \rightarrow \quad 1231231245 \\
& \begin{array}{l}
\text { column word of } \\
\text { cos }
\end{array}
\end{aligned}
$$

or

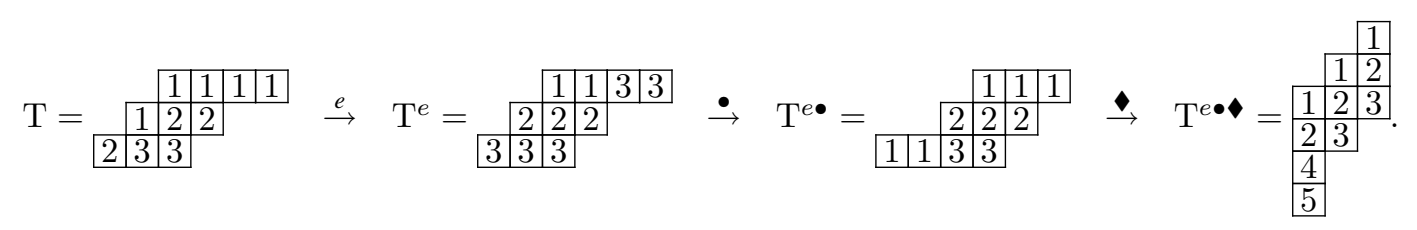

\section{Computational complexity of bijection $\downarrow$ and reduction of con- jugation symmetry map}

We show that the computational complexity of bijection is linear on the input. We follow closely ( $\mathrm{PV} 2)$ for this section. Using ideas and techniques of Theoretical Computer Science, see (AHU, CLRS), each bijection can be seen as an algorithm having one type of combinatorial objects as input, and another as output. We define a correspondence as an one-to-one map established by a bijection; therefore, obviously several different defined bijections can produce the same correspondence. In this way one can think of a correspondence as a function which is computed by the algorithm, viz. the bijection. The computational complexity is, roughly, the number of steps in the bijection. Two bijections are identical if and only if they define the same correspondence. Obviously one task can be performed by several different algorithms, each one having its own computational complexity, see (AHU, CLRS). For example we recall that there are several ways to multiply large integers, from naive algorithms, e.g. the Russian peasant algorithm, to that ones using FFT (Fast Fourier Transform), e.g. Schönhage-Strassen algorithm; see e.g. (GG) for a comprehensive and update reference. Formally, a function $f$ reduces linearly to $g$, if it is possible to compute $f$ in time linear in the time it takes to compute $g ; f$ and $g$ are linearly equivalent if $f$ reduces linearly to $g$ and vice versa. This defines an equivalence relation on functions, which can be translated into a linear equivalence on bijections.

Let $D=\left(d_{1}, \ldots, d_{n}\right)$ be an array of integers, and let $m=m(D):=\max _{i} d_{i}$. The bit-size of $D$, denoted by $\langle D\rangle$, is the amount of space required to store $D$; for simplicity from now on we assume that $\langle D\rangle=n\left\lceil\log _{2} m+1\right\rceil$. We view a bijection $\delta: \mathcal{A} \longrightarrow \mathcal{B}$ as an algorithm which inputs $A \in \mathcal{A}$ and outputs $B=\delta(A) \in \mathcal{B}$. We need to present Young tableaux as arrays of integers so that we can store them and compute their bit-size. Suppose $A \in Y T(\lambda / \mu ; m)$ : a way to encode $A$ is through its recording matrix $\left(c_{i, j}\right)$, which is defined by $c_{i, j}=a_{i, j}-a_{i, j-1}$; in other words, $c_{i, j}$ is the number of $j$ 's in the $i$-th row of $A$; this is the way Young tableaux will be presented in the input and output of the algorithms. Finally, we say that a map $\gamma: \mathcal{A} \longrightarrow \mathcal{B}$ is size-neutral if the ratio $\frac{\langle\gamma(A)\rangle}{\langle A\rangle}$ is bounded for 
all $A \in \mathcal{A}$. Throughout the paper we consider only size-neutral maps, so we can investigate the linear equivalence of maps comparing them by the number of times other maps are used, without be bothered by the timing. In fact, if we drop the condition of being size-neutral, it can happen that a map increases the bit-size of combinatorial objects, when it transforms the input into the output, and this affects the timing of its subsequent applications. Let $\mathcal{A}$ and $\mathcal{B}$ be two possibly infinite sets of finite integer arrays, and let $\delta: \mathcal{A} \longrightarrow \mathcal{B}$ be an explicit map between them. We say that $\delta$ has linear cost if $\delta$ computes $\delta(A) \in \mathcal{B}$ in linear time $O(\langle A\rangle)$ for all $A \in \mathcal{A}$. There are many ways to construct new bijections out of existing ones: we call such algorithms circuits and we define below several of them that we need.

○ Suppose $\delta_{1}: \mathcal{A}_{1} \longrightarrow \mathcal{X}_{1}, \gamma: \mathcal{X}_{1} \longrightarrow \mathcal{X}_{2}$ and $\delta_{2}: \mathcal{X}_{2} \longrightarrow \mathcal{B}$, such that $\delta_{1}$ and $\delta_{2}$ have linear cost, and consider $\chi=\delta_{2} \circ \gamma \circ \delta_{1}: \mathcal{A} \longrightarrow \mathcal{B}$. We call this circuit trivial and denote it by $I\left(\delta_{1}, \gamma, \delta_{2}\right)$.

$\circ$ Suppose $\gamma_{1}: \mathcal{A} \longrightarrow \mathcal{X}$ and $\gamma_{2}: \mathcal{X} \longrightarrow \mathcal{B}$, and let $\chi=\gamma_{2} \circ \gamma_{1}: \mathcal{A} \longrightarrow \mathcal{B}$. We call this circuit sequential and denote it by $S\left(\gamma_{1}, \gamma_{2}\right)$.

$\circ$ Suppose $\delta_{1}: \mathcal{A} \longrightarrow \mathcal{X}_{1} \times \mathcal{X}_{2}, \gamma_{1}: \mathcal{X}_{1} \longrightarrow \mathcal{Y}_{1}, \gamma_{2}: \mathcal{X}_{2} \longrightarrow \mathcal{Y}_{2}$, and $\delta_{1}: \mathcal{Y}_{1} \times \mathcal{Y}_{2} \longrightarrow \mathcal{B}$, such that $\delta_{1}$ and $\delta_{1}$ have linear cost. Consider $\chi=\delta_{2} \circ\left(\gamma_{1} \times \gamma_{2}\right) \circ \delta_{1}: \mathcal{A} \longrightarrow \mathcal{B}$ : we call this circuit parallel and denote it by $P\left(\delta_{1}, \gamma_{1}, \gamma_{2}, \delta_{2}\right)$.

For a fixed bijection $\alpha$, we say that $\beth$ is an $\alpha$-based ps-circuit if one of the following holds:

$\bullet \beth=\delta$, where $\delta$ is a bijection having linear cost.

$\bullet \beth=I\left(\delta_{1}, \alpha, \delta_{2}\right)$, where $\delta_{1}, \delta_{2}$ are bijections having linear cost.

- $\beth=P\left(\delta_{1}, \gamma_{1}, \gamma_{2}, \delta_{2}\right)$, where $\gamma_{1}, \gamma_{2}$ are $\alpha$-based ps-circuits and $\delta_{1}, \delta_{2}$ are bijections having linear cost.

$\bullet \beth=S\left(\gamma_{1}, \gamma_{2}\right)$, where $\gamma_{1}, \gamma_{2}$ are $\alpha$-based ps-circuits.

In other words, $\beth$ is an $\alpha$-based ps-circuit if there is a parallel-sequential algorithm which uses only a finite number of linear cost maps and a finite number of application of map $\alpha$. The $\alpha$-cost of $\beth$ is the number of times the map $\alpha$ is used; we denote it by $s(\beth)$.

Let $\gamma: \mathcal{A} \longrightarrow \mathcal{B}$ be a map produced by the $\alpha$-based ps-circuit $\beth$. We say that $\beth$ computes $\gamma$ at $\operatorname{cost} s(\beth)$ of $\alpha$. A map $\beta$ is linearly reducible to $\alpha$, write $\beta \hookrightarrow \alpha$, if there exist a finite $\alpha$-based ps-circuit $\beth$ which computes $\beta$. In this case we say that $\beta$ can be computed in at most $s(\beth) \operatorname{cost}$ of $\alpha$. We say that maps $\alpha$ and $\beta$ are linearly equivalent, write $\alpha \sim \beta$, if $\alpha$ is linearly reducible to $\beta$, and $\beta$ is linearly reducible to $\alpha$. We recall, gluing together, results proved in Section 4.2 of (PV2).

Proposition 4.1 Suppose $\alpha_{1} \hookrightarrow \alpha_{2}$ and $\alpha_{2} \hookrightarrow \alpha_{3}$, then $\alpha_{1} \hookrightarrow \alpha_{3}$. Moreover, if $\alpha_{1}$ can be computed in at most $s_{1}$ cost of $\alpha_{2}$, and $\alpha_{2}$ can be computed in at most $s_{2}$ cost of $\alpha_{3}$, then $\alpha_{1}$ can be computed in at most $s_{1} s_{2}$ cost of $\alpha_{3}$. Suppose $\alpha_{1} \sim \alpha_{2}$ and $\alpha_{2} \sim \alpha_{3}$, then $\alpha_{1} \sim \alpha_{3}$ Suppose $\alpha_{1} \hookrightarrow \alpha_{2} \hookrightarrow \ldots \hookrightarrow \alpha_{n} \hookrightarrow \alpha_{1}$, then $\alpha_{1} \sim \alpha_{2} \sim \ldots \sim \alpha_{n} \sim \alpha_{1}$.

We state now the computational complexity of bijection $\downarrow$ and the reduction of conjugation symmetry map. 
Algorithm 4.1 [Bijection $\$$.]

Input: $L R$ tableau $T$ of skew shape $\lambda / \mu$, with $\lambda=\left(\lambda_{1} \geq \ldots \geq \lambda_{n}\right)$,

$\mu=\left(\mu_{1} \geq \ldots \geq \mu_{n}\right)$, and filling $\nu=\left(\nu_{1} \geq \ldots \geq \nu_{n}\right)$, having $A=\left(a_{i, j}\right) \in M_{n \times n}(\mathbb{N}) \quad\left(a_{i, j}=0\right.$ if $j>i)$ as (lower triangular) recording matrix.

Write $\widetilde{A}$, a copy of the matrix $A$.

For $j:=$ n down to 2 do

For $i:=1$ to $n$ do

Begin

If $i=j$ then $\widetilde{a}_{i, i}:=\widetilde{a}_{i, i}+\lambda_{1}-\lambda_{i}$

else

End

If $j>i$ then $\widetilde{a}_{i, j}=0$ else $\widetilde{a}_{i, j}:=\widetilde{a}_{i, j}+\widetilde{a}_{i, j+1}$.

So far the computational cost is $O\left(n^{2}\right)=O(\langle A\rangle)$.

Set a matrix $B=\left(b_{i, j}\right) \in M_{\lambda_{1} \times \lambda_{1}}(\mathbb{N})$ such that $b_{i, j}=0$ for all $i, j$.

For $i:=1$ to $n$ do

Begin

Set $c:=0$.

For $j:=0$ to $n$ do

Begin

$r:=\widetilde{a}_{i+j, i}-a_{i+j, i}, \quad$ see Remark 4.2

For $t:=1$ to $a_{i+j, i}$ do $b_{r+t, c+t}:=b_{r+t, c+t}+1$.

$c:=c+a_{i+j, i}$

End

End

This part has total computational cost at most equal to

$$
O\left(\sum_{1 \leq i . j \leq n} a_{i, j}\right)=O(|\lambda \backslash \mu|)=O(|\lambda|-|\mu|)=O(\langle T\rangle) .
$$

Output: $B$ recording matrix of the output tableau.

Remark 4.2 For all $1 \leq i \leq n$ and $0 \leq j \leq n-i+1$, we have

$$
\widetilde{a}_{i+j+1, i}-\widetilde{a}_{i+j, i} \geq a_{i+j+1, i} .
$$

From Theorem 3.3 and this algorithm we have

Theorem 4.3 The conjugation symmetry maps $\varrho^{B S S}, \varrho^{W H S}$ and $\varrho_{3}$ are identical, and linear equivalent to the Schützenberger involution E,

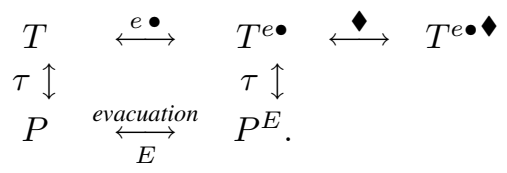

Thus conjugation symmetry maps and commutative symmetry maps are linearly reducible to each other. 
Acknowledgement: The first author wishes to thank Igor Pak and Ernesto Vallejo for their interest in the papers $(\mathrm{A} 1$; $\mathrm{A} 2)$.

\section{References}

[AHU] Alfred V. Aho, John E. Hopcroft, Jeffrey D. Ullman. The design and analysis of computer algorithms, Addison-Wesley Publishing Co., 1975.

[A1] Olga Azenhas. The admissible interval for the invariant factors of a product of matrices, Linear and Multilinear Algebra 46 (1999), 51-99.

[A2] Olga Azenhas. Littlewood-Richardson fillings and their symmetries, Matrices and group representations (Coimbra, 1998), 81-92, Textos Mat. Sér. B, 19, Univ. Coimbra, Coimbra, 1999.

[A3] Olga Azenhas. A variation on tableau switching and a Pak-Vallejo's Conjecture, DMTCS proc. AJ, 2008, 529-542. FPSAC /SFCA 2008, Talca University, Valparaiso, Chile, June 2008. Slides from the presentation available at

http://inst-mat.utalca.cl/fpsac2008/talks/Azenhas.pdf

[ACM] Olga Azenhas, Alessandro Conflitti, Ricardo Mamede. Identical bijections on the conjugation property of Littlewood-Richardson fillings, The 60th Séminaire Lotharingien de Combinatoire, Strobl, Austria. Slides from the presentation available at http: / / www.mat.univie.ac.at/ slc/

[BSS] Georgia Benkart, Frank Sottile, Jeffrey Stroomer. Tableau switching: algorithms and applications, J. Combin. Theory Ser. A 76 (1996), 11-34.

[BZ] Arkady Berenstein, Andrei Zelevinsky. Triple multiplicities for $s l(r+1)$ and the spectrum of the exterior algebra of the adjoint representation. J. Algebraic Combin. 1 (1992), 7-22.

[B] William H. Burge. Four correspondences betwen graphs and generalized Young tableaux, J. Combin. Theory Ser. A 17 (1974), 12-30.

[CLRS] Thomas H. Cormen, Charles E. Leiserson, Ronald L. Rivest, Clifford Stein, Introduction to Algorithms, The MIT Press, 2nd edition, 2001.

[DK1] Vladimir I. Danilov, Gleb A. Koshevoŭ. Massifs and the combinatorics of Young tableaux, Uspekhi Mat. Nauk 60 (2005), 79-142 (Russian); translation in Russian Math. Surveys 60 (2005), 269-334.

[DK2] Vladimir I. Danilov and Gleb A. Koshevol̆. Arrays and the octahedron recurrence, available at http://arxiv.org/abs/math/0504299

[F] William Fulton. Young Tableaux: With Applications to Representation Theory and Geometry, London Mathematical Society Student Texts, vol. 35, Cambridge University Press, Cambridge, 1997.

[GG] Joachim von zur Gathen, Jürgen Gerhard. Modern Computer Algebra, 2nd ed., Cambridge University Press, Cambridge, 2003. 
[GP] Oleg Gleizer, Alexander Postnikov. Littlewood-Richardson coefficients via Yang-Baxter Equation, Internat. Math. Res. Notices (2000), no. 14, 741-774.

[H] Mark Haiman. Dual equivalence with applications, including a conjecture of Proctor, Discrete Math. 99 (1992), 79-113.

[HS] Philip Hanlon, Sheila Sundaram. On a bijection between Littlewood-Richardson fillings of conjugate shape, J. Combin. Theory Ser. A 60 (1992), 1-18.

[K1] Ronald C. King. Littlewood-Richardson coefficients and the hive model, available at http://www.personal.soton.ac.uk/rck/seoul.pdf

[K2] Ronald C. King. Littlewood-Richardson coefficients, the hive model and Horn Inequalities, available at http://www.personal.soton.ac.uk/rck/coimbra.pdf

[KT] Allen Knutson, Terence Tao. The honeycomb model of $G \operatorname{Ln}(C)$ tensor products. I: Proof of the saturation conjecture, J. Amer. Math. Soc. 12 (1999) 1055-1090.

[KTW] Allen Knutson, Terence Tao, Christopher Woodward. The honeycomb model of $G L_{n}(\mathbb{C})$ Tensor products II: Puzzles determine facets of the Littlewood-Richardson cone, J. Amer. Math. Soc. 17 (2004), 19-48.

[Loth] Alain Lascoux, Bernard Leclerc, Jean-Yves Thibon. The plactic monoid in M. Lothaire (ed.), Algebraic Combinatorics on Words, Vol. 90 of Enciclopedia of Mathematics and its Applications, pp. 164-196, Cambridge University Press, Cambridge, United Kingdom, 2002.

[LS] Alain Lascoux, Marcel-Paul Schützenberger, Le monoïde plaxique, Noncommutative Structures in Algebra and Geometric Combinatorics, (Naples, 1978), Quad. "Ricerca Sci.", vol. 109, CNR, Rome (1981).

[Lee1] Mark A. A. van Leeuwen. Tableau algorithms defined naturally for pictures, Discrete Math. 157 (1996), 321-362.

[Lee2] Mark A. A. van Leeuwen. The Littlewood-Richardson rule and related combinatorics, in Interaction of combinatorics and representation theory, 95-145, MSJ Mem., 11, Math. Soc. Japan, Tokyo, 2001.

[LiRi] Dudley E. Littlewood, Archibald R. Richardson. Group characters and algebra, Philos. Trans. Roy. Soc. London Ser. A 233 (1934), 99-142.

[Mac] Ian G. Macdonald. Symmetric Functions and Hall Polynomials, Clarendon Press, Oxford University Press, 1995.

[O] Erik Ouchterlony. Commutation for Young tableau involutions, Linköping University, Phd Thesis, 2005.

[P] Igor Pak. Partition bijections, a survey, Ramanujan J. 12 (2006), 5-75.

[PV1] Igor Pak, Ernesto Vallejo. Combinatorics and geometry of Littlewood-Richardson cones, Europ. J. Combinat., 26 (2005), 995-1008. 
[PV2] Igor Pak, Ernesto Vallejo. Reductions of Young tableau bijections, to appear in SIAM J. Discrete Math. Available at http://www.arXiv:math/0408171 and http://www.math.mit.edu/ pak/tab7.pdf

[Pu] Kevin Purbhoo. Puzzles, tableaux, and mosaics, J. Algebraic Combin., 28 (2008), 461-480.

[Sa] Bruce Sagan. The Symmetric Group. Representations, Combinatorial Algorithms, and Symmetric functions, Springer Verlag, New York, 2001.

[Sch] Marcel-Paul Schützenberger. Quelques remarques sur une construction de Schensted, Math. Scandinavica 12 (1963), 117-128.

[St] Richard Stanley. Enumerative Combinatorics, Vol 2, Cambridge University Press, Cambridge, United Kingdom, 2001.

[Th] Glânffrwd P. Thomas. On a construction of Schützenberger, Discrete Math. 17 (1977), 107-118.

[TY] Hugh Thomas, Alexander Yong. An $S_{3}$-symmetric Littlewood-Richardson rule, Math. Res. Lett. 15 (2008), no. 5, 1027-1037.

[W] Dennis White. Hybrid tableaux and the Littlewood-Richardson rule, Discrete Math. 80 (1990), 183-206.

[Z] Ion Zaballa. Increasing and Decreasing Littlewood-Richardson Sequences and Duality, preprint, University of Basque Country, 1996. 
